\section{G234(P) CASE REPORT: PREMATURE ADRENARCHE AND PSEUDOHYPOPARATHYROIDISM - MECHANISTICALLY LINKED OR COINCIDENCE?}

${ }^{1} \mathrm{JC}$ Odone, ${ }^{1} \mathrm{Y}$ Kumar, ${ }^{2} \mathrm{CP}$ Burren. ${ }^{1}$ Paediatrics, Royal Cornwall Hospital, Truro, UK; ${ }^{2}$ Paediatric Endocrinology and Diabetes, Bristol Royal Hospital for Children, Bristol, UK

\subsection{6/archdischild-2018-repch.228}

Aims To describe a case of premature adrenarche with pseudohypoparathyroidism, an as yet unreported combination.

Methods An otherwise well 8 year old girl presented to a Paediatric Endocrine Clinic with early pubic hair development suggestive of premature adrenarche. Blood tests revealed hypocalcaemia, elevated phosphate and highly elevated parathyroid hormone $(\mathrm{PTH})$ level, giving a biochemical diagnosis of pseudohypoparathyroidism. She had normal stature (height 50th 75th centile) and no phenotypic features of Albright Hereditary Osteodystrophy (AHO) were identified: obesity, learning difficulties, brachydactyly, short stature, shortened 4th/5th metacarpals, dental hypoplasia or a rounded face.

Results Blood tests revealed low corrected calcium $1.49 \mathrm{mmol} / \mathrm{L}$ (reference range 2.2-5.7), elevated phosphate $2.78 \mathrm{mmol} / \mathrm{L}$ (reference range 0.9-1.8) and serum PTH level almost 10 times the upper limit of normal at $66.4 \mathrm{pmol} / \mathrm{L}$ (reference range 1.6-6.9), with normal Vitamin D $94 \mathrm{nmol} / \mathrm{L}$, normal thyroid function: Free T4 $5.4 \mathrm{pmol} / \mathrm{L}$ (reference range 12-22); TSH $4.8 \mathrm{miu} / \mathrm{L}$ (reference range $0.27-4.2$ ).

Hand and wrist Xray for bone age assessment revealed mildly shortened 4th $/ 5$ th metacarpals, a phenotypic feature of AHO. Genetic testing results and MRI head to screen for white matter calcification are awaited. These will help clarify which subtype of pseudohypoparathyroidism is responsible for this presentation.

Management She commenced oral calcium carbonate and alfacalcidol to correct the severe calcium deficiency and to normalise PTH levels. Progressively increasing doses have been required.

Conclusion Pseudohypoparathyroidism is a rare endocrine disorder characterised by resistance to the action of PTH. It has been classified within the AHO group. Recognition of a broader range of phenotypic features and underlying mutations has led to a novel classification system of iPPSD (inactivating PTH/PTHrP signalling disorders) developed by the EuroPHP network. GNAS1 mutations have been identified underlying various pseudohypoparathyroidism subtypes, resulting in reduced function of the G-protein coupled to the PTH receptor. G-proteins are also coupled to other hormone receptors; patients with AHO or iPPSD often present with a range of endocrine disorders, for example hypothyroidism. There are cases of individuals with GNAS1 mutations presenting concurrently with precocious puberty and pseudohypoparathyroidism but no reported case of premature adrenarche and pseudohypoparathyroidism. Awaited genetic studies in our case may be informative.

\section{G235(P) HBA1C CONTROL IN TYPE 1 DIABETES WITH RETINOPATHY, A REASSESSMENT AT 5 YEARS}

CA Sherlaw, S Karandikar, J Rweyemamu. Paediatrics, Heartlands Hospital, Birmingham, UK

10.1136/archdischild-2018-rcpch.229

Aims The National Screening Committee and NICE recommend monitoring of diabetes patients for retinopathy from the age of 12 years as early treatment is known to improve outcomes. Control of HbA1c may reverse background retinopathy and good control of blood pressure can delay diabetic retinopathy by $4-5$ years, monitoring blood pressure has therefore been included in NICE guidance. We firstly aimed to assess compliance with NICE guidelines at Heartlands Hospital in 2015-2016 compared to results from 2011. Secondly we aimed to compare HbA1c levels in those with retinopathy compared to those without.

Methods Review of the electronic database of patients known to the diabetes team at Heartlands Hospital between 1 st April 2015 and 31 st March 2016. The inclusion criteria were those with type 1 diabetes mellitus and age over 12 years by 31 st March 2016.

Results 137 patients met the inclusion criteria of whom $81.6 \%$ had been screened for retinopathy, this is compared to $68 \%$ in 2011. Of those screened $18 \%$ had background retinopathy and $2 \%$ preproliferative retinopathy with the remainder normal. Mean HbA1c for those without retinopathy $(80.51 \mathrm{mmol} / \mathrm{mol})$ was lower than in those with background retinopathy $(91.05 \mathrm{mmol} / \mathrm{mol})$ and preproliferative retinopathy (100 mmol/mol). These HbA1c results were lower than 2011 when mean $\mathrm{HbA} 1 \mathrm{c}$ was $83.6 \mathrm{mmol} / \mathrm{mol}$ for those without retinopathy and $108.7 \mathrm{mmol} / \mathrm{mol}$ in those with background retinopathy. Despite this improvement, HbA1c is higher in our cohort than the national mean. Annual blood pressure check had been performed in $99.3 \%$ of patients.

Conclusion Higher HbA1c in those with retinopathy is consistent with the literature. While there has been an improvement in both rates of retinopathy screening and HbA1c levels between 2011 and 2016 at Heartlands hospital, rates of retinopathy overall remain similar. There has however been an improvement in average $\mathrm{HbA} 1 \mathrm{c}$ although this is still higher than national average, this may reflect the deprived population served by the hospital. Those who had failed to attend screening were identified to ensure further education.

\section{G236(P) ANALYSING THE INCIDENCE OF CONGENITAL HYPOTHYROIDISM IN THE LOCAL AREA}

RS Tattersall, R Nicholls, Y Kumar. Paediatrics, Royal Cornwall Hospitals NHS Trust, Truro, UK

\subsection{6/archdischild-2018-rcpch.230}

Aims This study aims to establish the incidence of congenital hypothyroidism $(\mathrm{CH})$ and the sub-type most prevalent in the local area. A secondary aim is to assess the correlation between the dosage of thyroxine replacement in dyshormonogenesis (CHD) and recovery potential. Furthermore, it will look for any link between Down syndrome and $\mathrm{CH}$.

Method Data from patients diagnosed with $\mathrm{CH}$ over a 16 year period was analysed to find the incidence, type of $\mathrm{CH}$ and, if $\mathrm{CHD}$, dose of leveothyroxine and outcome of a recovery test if undertaken. Whether there were any associated co-morbidities was also recorded.

Results From 55 patients, 50 originally from the local area, 47 were analysed, with full records not available for the rest. The most prevalent form was CHD with 22 cases followed by lingual thyroid in 13 . The dose of levothyroxine in CHD was variable, ranging from $25 \mathrm{mcg}$ to $200 \mathrm{mcg}$ daily. A few patients with CHD were too young for the recovery test $(8$ patients) but, of the 14 patients offered the test, only 8 
accepted. 6 of these 8 patients showed full recovery of thyroid function, with a lower levothyroxine dose evident in 5 cases. 2 of these patients had associated co-morbidities Down syndrome and Deletion 1q21.1 syndrome. Overall, 4 patients with $\mathrm{CHD}$ had associated co-morbidities, whereas no other forms of $\mathrm{CH}$ had any.

Conclusion The incidence of $\mathrm{CH}$ in the local area is approximately 1 in 1200, much higher than the UK average of 1 in 4000. Dyshormonogenesis accounts for the greatest proportion of $\mathrm{CH}(40 \%)$ compared to established literature where it is shown to be $10 \%-20 \%$. Further investigation, therefore, is required to see why $\mathrm{CHD}$ is more common in this area. There is insufficient information available to draw conclusions with regards to likelihood of recovery. However, the study is suggestive of recovery being more likely with a lower dose of levothyroxine, and for those patients with associated syndromes. In order to test this hypothesis, a larger study needs to be undertaken.

\section{G237(P) EFFECTIVENESS OF THE SENSOR AUGMENTED PUMP IN MANAGING TYPE I DIABETES MELLITUS}

S Kattera, JM Robinson, JOoi Enn. Child Health, Wrightington, Wigan and Leigh NHS Foundation Trust, Manchester, UK

\subsection{6/archdischild-2018-rcpch.231}

Aim Type I Diabetes Mellitus is a chronic metabolic disorder with an incidence of 24000 children in the UK. NICE has recommended the use of the sensor augmented pump (SAP) in managing short and long term diabetes related complications. Limited literature is available in the paediatric population. SAP is designed to allow continuous glucose monitoring, with realtime adjustment of insulin; making it superior to other pump systems. SAP was assessed in a trial of children; that were previously on insulin pumps with limited success in managing hypoglycaemic episodes. The aim of the audit was to identify how effective SAP was in reducing HBA1c and preventing hypoglycaemic episodes.

Method 14 children were commenced on SAP between 20162017 March. Evidence was collected from electronic records; which, summarised clinic letters from both the consultant and diabetic nurse team. Date of diagnosis and initiation of SAP following that was dictated. The number of hypoglycaemic episodes causing admission to hospital and HBA1c was compared before and after SAP was started.

Results There were equal number of males and females. The average age of Type I DM diagnosis was 6 years 2 months. The average age when SAP was commenced was 8 years 1 month.

Average HBA1c prior to SAP was 63.1 and showed 5.3\% improvement following SAP with 59.8. 21.4\% of children showed deterioration in HBA1c. These were males and belonged to an older age group ( $>5$ years). $14.3 \%$ of children did not show any change in their HBA1c.

$29 \%$ of children had a severe hypoglycaemic episode prior to SAP. Following SAP, 7.1\% of children had a severe hypoglycaemic episode.

Conclusion NICE 2016 has recommended the use of SAP in improving the quality of life for people with Type I DM. The audit highlighted improvement in glucose control with positive effect on HBA1c and reduction in hypoglycaemic episodes. This can be explained with increased adherence to treatment with the use of automatic insulin monitoring and delivery. Ultimately, NHS resource and cost saving is achieved; with the quicker target blood glucose and reduced hospital admissions related to Type I DM disease complications.

\section{G238(P) ABSTRACT WITHDRAWN}

\section{G239(P) A REVIEW OF CHILDREN PRESENTING WITH A NEW DIAGNOSIS OF DIABETES}

CS Cockburn, B Rayen. Paediatic Services, Dumfries and Galloway Royal Infirmary, Dumfries, UK

\subsection{6/archdischild-2018-rcpch.232}

Introduction Diabetic ketoacidosis (DKA) can be a life threatening presentation of Type 1 diabetes in children and young people. Data had previously been collected (from January 2007 - August 2009) that demonstrated that 30\% of newly diagnosed diabetics presented in DKA. Following these results and using the Parma study, a public health awareness campaign was completed using $(\mathrm{P}) \mathrm{s}$ designed by local school children to try and improve public awareness around presentations of diabetes.

Aims To review whether children presented in DKA as their first diagnosis of Type 1 diabetes and whether they had presented to health services on more than one occasion prior to diagnosis.

Methods A list of patients was collected from the Diabetic Team caseload and SCI diabetes database to identify children and young people diagnosed between January 2014 and July 2017. Case notes were retrospectively reviewed using a proforma that was developed in consultation with the diabetes team. 33 patients were included.

Results The majority of patients continued to be diagnosed by GP $(58 \%)$, while out of hours $(\mathrm{OOH})$ made $18 \%$ of diagnoses, $12 \%$ were made by the emergency department (ED) and $12 \%$ by paediatrics. Previously $55 \%$ of diagnoses were made by the GP, $10 \%$ by ED and $20 \%$ by paediatrics. Six of the thirty three patients presented in DKA $(19 \%)$ with the remaining $81 \%$ (27/33) presenting 'walking wounded'. In comparison to the previous data collection which had demonstrated $30 \%$ (6 of 20) presented with DKA. $42 \%$ of patients were diagnosed at their first presentation, with a further $21 \%$ on their second presentation, $9 \%$ presented on three or more occasions before they were diagnosed.

Conclusion There has been a reduction in percentage of new diabetic children presenting in DKA to services. This may suggest a greater awareness in the community and could be related to a previous public health campaign. It is still concerning that almost 20\% (1 in 5) new diabetics present with DKA and that only $42 \%$ of patients were diagnosed at initial presentation. On some occasions diabetes was suspected but the patients were asked to return with a urine sample before referral to paediatric services was made. 